\title{
DETERMINAÇÃO DA EVAPOTRANSPIRAÇÃO POR DIFERENTES MÉTODOS E SUA APLICAÇÃO NO ÍNDICE DE SECA NA CAMPANHA GAÚCHA, BRASIL ${ }^{1}$
}

\author{
GABRIEL FRANKE BRIXNER ${ }^{2}$, EDGAR RICARDO SCHÖFFEL ${ }^{3}$, JORGE TONIETTO ${ }^{4}$
}

RESUMO - O desempenho dos métodos para estimar a evapotranspiração de referência (ETo) varia em função das condições climáticas locais. Por isso, este trabalho tem como objetivos: a) avaliar o desempenho dos métodos indiretos de cálculo da ETo, na escala diária e mensal, para o período de outubro a março; b) avaliar os métodos para uso em estudos de zoneamento vitícola no cálculo do Índice de Seca (IS); ambos tendo como método-padrão Penman-Monteith-FAO. Foram utilizados dados meteorológicos diários dos meses de outubro a março, de 1961 a 2010, dos postos meteorológicos da rede da FEPAGRO e do INMET, localizados na região da Campanha-RS. A ETo foi calculada pelos métodos de Thornthwaite, Camargo, Makkink, Radiação Solar, Jensen-Haise, Linacre, Hargreaves-Samani, Blaney-Criddle e Penman-Monteith, com posterior aplicação ao IS, comparando aos resultados obtidos no método-padrão. Verifica-se que, na ETo na escala diária, ocorrem diferenças quanto ao desempenho entre os métodos, variando a classificação de "sofrível" a "muito bom". Na escala mensal, os métodos têm o melhor desempenho, apresentam classificação como "bom", para os métodos de Radiação Solar, Makkink, Camargo e Blaney-Criddle. Para o IS, no mês de março, verificou-se que os métodos de Thornthwaite e Camargo apresentam desempenho "ótimo", sendo, portanto, metodologias recomendadas para a estimativa da ETo no Sistema de Classificação Climática Multicritério (CCM), para a Campanha-RS.

Termos para indexação: Penman-Monteith, métodos empíricos, evapotranspiração de referência, Sistema de Classificação Climática Multicritério (CCM) Geovitícola.

\section{DETERMINATION OF THE EVAPOTRANSPIRATION BY DIFFERENT METHODS AND ITS APPLICATION IN THE DRYNESS INDEX IN THE CAMPANHA GAUCHA REGION, BRAZIL}

\begin{abstract}
The performance of the methods for estimating reference evapotranspiration (ETo) varies depending on the local climate. Therefore, this study aimed to: a) assess the performance of the indirect method of ETo estimation, on the daily and monthly scale, for the period from October to March, b) verify the quality of the methods for use in viticultural zoning studies for calculation of Dryness Index (DI), both having as standard method the Penman-Monteith-FAO. Daily meteorological data were used from October to March, in 1961 to 2010 period, from the network of meteorological stations FEPAGRO and INMET located in Campanha, RS. The evapotranspiration was calculated by the methods of Thornthwaite, Camargo, Makkink, Solar Radiation, Jensen-Haise, Linacre, Hargreaves-Samani, Blaney-Criddle and Penman-Monteith, later determined the DI, comparing them with those obtained by Penman-Monteith. It was found on the daily scale that ETo showed differences in performance between the methods, varying the classification of "poorly" to "very good". On a monthly scale, methods that performed best had a performance rated as "good," for methods of Solar Radiation, Makkink, Blaney-Criddle and Camargo. For the dryness index in March, it turns out that the methods of Thornthwaite and Camargo showed great performance, being methodologies that can be adopted to estimate evapotranspiration for DI in the Geoviticulture Multicriteria Climatic Classification System for Campanha, RS.

Index terms: Penman-Monteith, empirical methods, reference of evapotranspiration, Geoviticulture Multicriteria Climatic Classification (MCC) System.

${ }^{1}$ (Trabalho 381-13). Recebido em: 08-10-2013. Aceito para publicação em: 30-04-2014. O presente trabalho é parte da Dissertação de Mestrado, defendido pelo primeiro autor, no PPG em Sistemas de Produção Agrícola Familiar (SPAF), da Universidade Federal de Pelotas (UFPel).

${ }^{2}$ Eng. Agrônomo, doutorando do PPGA/UFSM, Santa Maria - RS. E-mail: brixner_gfb@yahoo.com.br

${ }^{3}$ Eng. Agrônomo, Dr. Professor da Faculdade de Agronomia Eliseu Maciel (FAEM/UFPel), Pelotas - RS. E-mail: ricardo_schoffel@ ufpel.edu.br

${ }^{4}$ Eng. Agrônomo, Dr. Pesquisador da Embrapa Uva e Vinho, Bento Gonçalves - RS. E-mail: jorge.tonietto@embrapa.br
\end{abstract}




\section{INTRODUÇÃO}

As principais regiões produtoras de uvas para vinhos finos no Sul do Brasil são as regiões da Serra Gaúcha, Campanha, Serra do Sudeste no Rio Grande do Sul e Planalto Catarinense em Santa Catarina. A vitivinicultura na Campanha teve início no fim dos anos de 1970. Nos anos 2000, a região ganhou destaque pelo incremento na área plantada, com crescente vinificação dos vinhos finos elaborados na própria região. Desta forma, a vitivinicultura tornouse uma alternativa para gerar desenvolvimento econômico para a Metade Sul, do Rio Grande do Sul (RATHMANN et al., 2008; AMARAL et al., 2009; PÖTTER et al., 2010).

Como a introdução da viticultura é recente na Campanha, tornam-se importantes estudos detalhados de zoneamento climático, contribuindo para a exploração adequada do potencial do meio vitícola. Dentre as metodologias que relacionam as condições climáticas à produção vitícola e ao potencial qualitativo das uvas destinadas à vinificação, destaca-se a metodologia do Sistema Classificação Climática Multicritério Geovitícola (Sistema CCM Geovitícola), proposto por Tonietto e Carbonneau (2004). Ela foi utilizada na caracterização climática das regiões vitivinícolas Iberoamericanas (TONIETTO; FIALHO, 2012), sendo uma metodologia de referência internacional para zoneamento climático vitivinícola, integrante da Resolução OIV-VITI 423-2012.

Esta metodologia permite o tratamento da informação climática de interesse vitícola na escala mundial (TONIETTO; CARBONNEAU, 2004), sendo composta por três índices: Índice Heliotérmico (IH), que quantifica a disponibilidade térmica do clima no ciclo vegetativo da videira, por meio da temperatura média e máxima mensal; Índice de Frio Noturno (IF), que relaciona as condições nictotérmicas de maturação da uva, por meio da temperatura mínima deste período; e Índice de Seca (IS), que corresponde ao balanço hídrico para cultura da videira no período vegetativo, utilizando dados de precipitação e da evapotranspiração de referência (ETo) mensal.

O cálculo do IS no Sistema CCM utiliza o método de Penman-Monteith-FAO como método-padrão para estimar a ETo. Este método micrometeorológico é considerado, pela comunidade científica mundial, o mais apropriado, pois considera os fenômenos biofísicos da evapotranspiração, utilizando um conjunto importante de variáveis meteorológicas, medidas em estações meteorológicas convencionais (CARVALHO et al., 2011).
Contudo, muitas regiões vitícolas do mundo não dispõem da medição de todas as variáveis meteorológicos necessárias para estimar a ETo pelo método recomendado pelo Sistema CCM Geovitícola. Tornando-se importante o uso de métodos alternativos para determinação da ETo, que utilizem variáveis meteorológicas mais comumente disponíveis. Isso possibilita a caracterização da demanda hídrica mesmo a partir de uma base mais limitada de dados meteorológicos (CONCEIÇÃO et al., 2012).

Existem diferentes métodos para calcular a ETo que utilizam um ou mais elemento meteorológicas, tais como: temperatura do ar, radiação solar, umidade relativa do ar e vento. No entanto, Pereira et al. (2009) observam que, antes de aplicar um método para determinado local ou região, é necessário verificar o desempenho deste em relação ao método-padrão, em diferentes escalas de tempo e, quando necessário, fazer calibrações a fim de minimizar erros de estimativa.

Segundo Carvalho et al. (2011), dentre os métodos mais simples de cálculo da ETo tem destaque o de Thornthwaite, que utiliza somente dados de temperatura do ar e do fotoperíodo local. Os mesmos autores destacam os métodos de Hargreaves e Samani, Jensen e Haise e Camargo por utilizarem dados de temperatura e radiação solar (extraterrestre e global). Dentre os métodos que utilizam maior número de variáveis meteorológicas, Carvalho et al. (2011) destacam o de Makkink, que necessita de dados de radiação solar global e temperatura de bulbo úmido, bem como os métodos da Radiação Solar e de Blaney-Criddle que, além da temperatura do ar, da temperatura de bulbo úmido e da radiação solar, necessitam, também, das medidas de vento, umidade relativa do ar, insolação e fotoperíodo. Já o método de Linacre, segundo os mesmos autores, necessita das informações de altitude, latitude, temperatura do ar e ponto de orvalho.

Quando se deseja comparar regiões vitícolas pelo IS, é importante a utilização da metodologiapadrão de cálculo da ETo, no Sistema CCM, para que não haja diferenças decorrentes da metodologia utilizada. Porém, quando necessário, métodos alternativos de cálculo da ETo podem ser utilizados, desde que seja testada sua similaridade em relação ao padrão (Penman-Monteith-FAO) para o clima da região (CONCEIÇÃO et al., 2012).

Diante disso, considerando as condições climáticas da região da Campanha do Rio Grande do Sul, o presente trabalho tem como objetivos: a) avaliar o desempenho dos métodos indiretos de cálculo da evapotranspiração de referência (ETo), 
na escala diária e mensal, para o período de outubro a março; b) avaliar os métodos para uso em estudos de zoneamento vitícola no cálculo do Índice de Seca (IS), ambos tendo como método-padrão PenmanMonteith-FAO.

\section{MATERIAL E MÉTODOS}

O estudo teve como área geográfica de abrangência a região da Campanha Gaúcha, no Estado do Rio Grande do Sul, conforme o macrozoneamento agroecológico e econômico do Estado do Rio Grande do Sul. A região é compreendida por 14 municípios, em duas sub-regiões: Fronteira Uruguai (Aceguá, Pedras Altas, Bagé, Candiota, Dom Pedrito, Herval, Hulha Negra, Quaraí e Santana do Livramento) e Uruguaiana/São Gabriel (Alegrete, Rosário do Sul, São Gabriel, Uruguaiana e Barra do Quaraí) (Figura 1).

Foram utilizados dados diários do período de 1961 a 2010, de seis estações meteorológicas localizadas na região da Campanha, conforme disponibilidade de dados discriminada na Figura 2.
Os dados meteorológicos foram obtidos por meio dos postos meteorológicos das redes do Instituto Nacional de Meteorologia (INMET) e da Fundação Estadual de Pesquisa Agropecuária no Rio Grande do Sul (FEPAGRO). Para os municípios que possuíam dois postos meteorológicos, utilizaram-se os dados coletados pelo INMET, e utilizados os da FEPAGRO para preencher eventuais dados faltantes na base de dados (Figura 2).

Os valores da evapotranspiração de referência (ETo) foram estimados diariamente, para o período de outubro a março, sendo que os valores mensais correspondem ao somatório da ETo diária. O estudo teve a abrangência destes meses por corresponderem aos meses associados ao ciclo vegetativo da videira (brotação-colheita), bem como por serem os mesmos utilizados para o cálculo do IS. Os métodos usados para calcular a ETo foram: Thornthwaite (ETo_TH) (1), Camargo (ETo CA) (2), Makkink (ETo MK) (3), Radiação Solar (ETo_RS) (4), Jensen-Haise (ETo_JH) (5), Linacre (ETo_LI) (6), HargreavesSamani (ETo_HS) (7), Blaney-Criddle (ETo_BC) (8) e Penman-Monteith (ETo PM) (9), calculados segundo as equações, respectivamente:

$$
\begin{aligned}
& \text { ETo_TH }=16\left(10 \frac{\mathrm{T}}{\mathrm{I}}\right)^{\mathrm{a}} 0 \leq \mathrm{T}<26,5^{\circ} \mathrm{C} \\
& \text { ETo_C } \Lambda=\text { F Q'oT ND } \\
& \text { ETo_MK }=(0,61 \mathrm{~W} \text { Rs }) \quad 0,12 \\
& \text { ETo_RS }=c_{o} \mid c_{1} \text { W Rs } \\
& \text { ETo_JH }=\operatorname{Rs}(0,0252 \mathrm{~T} \mid 0,078) \\
& \text { ETo_LI }=\frac{\frac{500(\mathrm{~T}+0,006 \mathrm{~h})}{(100-\varphi)}+15\left(\mathrm{~T}-\mathrm{T}_{0}\right)}{80-\mathrm{T}} \\
& \text { ETo_HS }=0,0023 \mathrm{Q}^{\prime} \mathrm{o}\left(\mathrm{T}_{\max } \mathrm{T}_{\min }\right)^{0,5}(\mathrm{~T} \mid 17,8) \\
& \text { ETo_BC }=a_{1} \mid \text { b p }(0,46 \mathrm{~T} \mid 8,13) \\
& \text { ETo_PM }=\frac{0,408 \mathrm{~s}(\mathrm{Rn}-\mathrm{G})+\frac{900 \gamma \mathrm{U}_{2}\left(\mathrm{e}_{\mathrm{s}}-\mathrm{e}_{2}\right)}{(\mathrm{T}+273)}}{(\mathrm{s}+\gamma)\left(1+0,34 \mathrm{U}_{2}\right)}
\end{aligned}
$$


em que: $\mathrm{T}=$ temperatura média do ar; $\mathrm{I}$ e a $=$ índices térmicos de cada região (Thornthwaite); Q'o = radiação solar extraterrestre diária; $\mathrm{ND}=$ número de dias do período; $\mathrm{F}$ = fator de ajuste variável com a temperatura; Rs = radiação solar global; $W=$ fator de ponderação em função da temperatura; c0 e c1 = coeficientes variáveis com a umidade relativa e a velocidade do vento; $\mathrm{h}=$ altitude; $\theta=$ latitude; To = temperatura do ponto de orvalho; Tmáx $=$ temperatura máxima; Tmín = temperatura mínima; a e b coeficientes da equação de Blaney Criddle; $\mathrm{p}=$ porcentagem do fotoperíodo anual; $\mathrm{Rn}=$ saldo diário de radiação; $\mathrm{G}=$ fluxo de calor no solo; $\mathrm{T}=$ temperatura média diária; U2 = velocidade média diária do vento medido a $2 \mathrm{~m}$ de altura; es = pressão de saturação de vapor; ea = pressão atual de vapor; $\mathrm{s}=$ declividade da curva de pressão de vapor; $\gamma=$ coeficiente psicométrico.

Conforme descrito por Liu et al. (2009), no método de Penman-Monteith, foi utilizada a metodologia recomendada pelo documento FAO 56 para estimar a pressão atmosférica, baseada na altitude, e para estimar a temperatura de bulbo úmido, baseada na umidade relativa média, quando não existia disponibilidade de tais variáveis na base de dados disponível. Para Alegrete, Quaraí e São Gabriel, a evapotranspiração de referência não foi estimada pelos métodos de Makkink e Radiação Solar devido à indisponibilidade de dados de temperatura de bulbo úmido no banco de dados meteorológicos destes municípios (Figura 2).

O fluxo de calor no solo $(G)$ foi calculado a partir da diferença entre a temperatura média diária $\left(\mathrm{T}_{\mathrm{md}}\right)$ e a temperatura média dos três dias anteriores $\left(\mathrm{T}_{3 \mathrm{md}}\right)$, pela seguinte expressão, descrita por Silva et al. (2011):

$$
G=0,38\left(T_{m d} \quad T_{3 m d}\right)
$$

O saldo de radiação $\left(\mathrm{MJ} \mathrm{m}^{-2} \mathrm{~d}^{-1}\right)$ foi calculado, conforme metodologia recomendada pelo documento FAO 56 e utilizado por Liu et al. (2009), Pereira et al. (2009) e Silva et al. (2011), somando-se o saldo de radiação de ondas curtas, estimado por meio da insolação, utilizando coeficientes calibrados mensalmente para a região da Campanha (FONTANA; OLIVEIRA, 1996), com o saldo de radiação de ondas longas $\left(\mathrm{MJ} \mathrm{m}^{-2} \mathrm{~d}^{-1}\right)$.

Após o cálculo da estimativa diária da evapotranspiração de referência, para cada método, foi feito o somatório mensal destes dados. Posteriormente, foi determinado o Índice de Seca (IS), segundo a metodologia de Tonietto e Carbonneau (2004):

$$
\mathrm{IS}=\sum_{\text {out }}^{\operatorname{mar}} \mathrm{Wo}+\mathrm{P}-\mathrm{Tv}-\mathrm{Es}
$$

em que: Wo é a reserva hídrica inicial do solo (200 mm); P é a precipitação mensal (mm); Tv é a transpiração potencial mensal do vinhedo (mm); Es é a evaporação potencial mensal do solo ( $\mathrm{mm}$ ). Para o cálculo da Tv, foi empregada a expressão:

\section{$\mathrm{Tv}=\mathrm{ETolk}$}

em que : $\mathrm{k}$ é o coeficiente de absorção de radiação pelas videiras, com valores equivalentes a 0,1 para outubro, considerado como o primeiro mês do ciclo vegetativo da cultura, 0,3 para novembro e 0,5 para dezembro, janeiro, fevereiro e março.

Es foi determinada pela expressão:

$\mathrm{Es}=\left(\frac{\mathrm{ETo}}{\mathrm{N}}\right)(1-\mathrm{k}) \mathrm{JPm}$

em que: N é o número de dias do mês, e JPm é o número de dias por mês de evaporação efetiva do solo, que é estimado dividindo-se $\mathrm{P}$ por cinco e que deve ser igual ou inferior a N. Os intervalos de classe para o Índice de Seca (IS, mm), com sua respectiva simbologia e intervalo de abrangência da classe, são indicados conforme proposto por Tonietto e Carbonneau (2004) (Tabela 1).

Após o cálculo da estimativa diária da evapotranspiração de referência para cada método e da determinação do Índice de Seca, foi determinado o coeficiente de determinação $\left(\mathrm{R}^{2}\right)$ e os índices de precisão (r), de exatidão (d) e de desempenho (c), obtidos da correlação linear entre cada método com o método-padrão de Penman-Monteith. A precisão foi avaliada pelo coeficiente de correlação (r) que indica o grau de dispersão dos dados obtidos em relação à média (BORGES JÚNIOR et al., 2012; PILAU et al., 2012), ou seja, o erro aleatório, o qual é determinado pela expressão:

$r=\frac{\sum\left[\left(x_{i}-\bar{x}_{1}\right)\left(y_{i}-\bar{y}_{1}\right)\right]}{\sqrt{\sum\left(x_{i} \quad \bar{x}_{1}\right)^{2} \sum\left(y_{i} \quad \overline{y_{1}}\right)^{2}}}$

em que: $x_{i}$ são os valores a serem comparados com o método-padrão; são os valores estimados pelo método-padrão e e são as médias respectivas de e .

A exatidão foi determinada pelo índice de Willmott (d), segundo metodologia apresentada por Camargo e Sentelhas (BORGES JÚNIOR et al., 2012; PILAU et al., 2012), expresso por: 
$\mathrm{d}=1-\left[\frac{\sum\left(\mathrm{x}_{1}-\mathrm{y}_{1}\right)^{2}}{\sum\left(\left|\mathrm{x}_{\mathrm{i}}-\overline{\mathrm{y}_{1}}\right|+\left|\mathrm{y}_{\mathrm{i}}-\overline{\mathrm{y}_{1}}\right|\right)^{2}}\right]$

O índice de desempenho (c), que resulta do produto entre os índices de precisão (r) e de exatidão (d), foi utilizado para classificar o desempenho de cada método de cálculo da ETo com o métodopadrão (ETo_PM), nas escalas diárias e mensal, para o período de outubro a março. Da mesma forma, foi determinado o desempenho dos métodos de cálculo da ETo aplicados ao IS com o método- padrão do CCM (ETo_PM), na escala mensal, para o mês de março.

A classificação quanto ao desempenho ocorreu com base na metodologia proposta por Camargo e Sentelhas (BORGES JÚNIOR et al., 2012; PILAU et al., 2012), da seguinte forma: ótimo, quando c apresenta valores maiores do que 0,86 ; muito bom, para valores entre 0,76 e 0,85 ; bom, para valores entre 0,66 e 0,75 ; mediano, para valores entre 0,61 e 0,65 ; sofrível, para valores entre 0,51 e 0,60 ; mau, para valores entre 0,41 e 0,50 ; e péssimo, para valores do índice c inferiores a 0,41 .

Para dar suporte a essa classificação, também foi calculado o valor do Erro-Padrão de Estimativa (EPE), na escala diária e mensal da ETo, para o período de outubro a março. Da mesma forma, foi determinado o Erro-Padrão da Estimativa aplicado ao Índice de Seca (EPE_IS), na escala mensal, para o período de outubro a março e para o mês de março, através da expressão:

$$
\text { EPE }=\sqrt{n-1 \sum_{i=1}^{n}\left(x_{i}-y_{i}\right)^{2}}
$$

\section{RESULTADOS E DISCUSSÃO}

As estimativas da evapotranspiração na escala diária pelos diferentes métodos, para o período de outubro a março, estão apresentadas na Tabela 2, e observou-se que, para os 50 anos, o método de Penman-Monteith apresentou, em média, 3,91 mm $\mathrm{d}^{-1}$, sendo que o valor máximo diário estimado chegou a $10,40 \mathrm{~mm} \mathrm{~d}^{-1}$, verificado na estação do verão.

Analisando os valores de ETo obtidos pelos demais métodos, foi possível destacar Linacre, Camargo, Thornthwaite e Blaney-Criddle, que apresentaram estimativas próximas das obtidas pelo método de Penman-Monteith. Por outro lado, com exceção de Blaney-Criddle, estes foram os métodos que apresentaram menor índice de precisão (r) e menor coeficiente determinação $\left(\mathrm{R}^{2}\right)$, com 0,51 ; 0,47 e 0,43 , respectivamente, com desempenho classificado como "sofrível" (SF) (Tabela 2). O método de Hargreaves-Samani também apresentou desempenho "sofrível", porém o valor médio do método $\left(5,15 \mathrm{~mm} \mathrm{~d}^{-1}\right)$ foi distante da estimativa ETo PM.

O baixo desempenho dos métodos de Linacre e de Thornthwaite pode ser explicado por serem metodologias desenvolvidas para estimar a evapotranspiração de referência mensal (CARVALHO et al., 2011), e não na escala diária, conforme calculado. O mesmo pode ser interpretado para o método de Camargo, cuja metodologia é baseada no método de Thornthwaite, sendo este recomendado para calcular a ETo nas escalas mensal ou decendial (CARVALHO et al., 2011).

Os métodos Radiação Solar, Jensen-Haise e Blaney-Criddle apresentaram desempenho considerado "bom", na escala diária (Tabela 2), com destaque para o método de Blaney-Criddle, que apresentou desempenho de 0,72 e erro-padrão da estimativa de $0,85 \mathrm{~mm} \mathrm{~d}^{-1}$. Entre todos os métodos avaliados, Makkink apresentou o melhor desempenho $(\mathrm{c}=78)$, com desempenho classificado como "muito bom", e EPE inferior a $1 \mathrm{~mm} \mathrm{~d}^{-1}$.

Em estudo desenvolvido por Pereira et al. (2009), na região da Serra da Mantiqueira (clima do tipo Cwb, segundo Köppen), com invernos frios e menos úmidos, com verões com temperaturas agradáveis e mais chuvosos, os métodos da Radiação Solar, de Jensen-Haise, de Blaney-Criddle e de Makkink também apresentaram os melhores desempenhos na escala diária, apesar da grande diferença climática da região da Serra da Mantiqueira e a Campanha (clima do tipo Cfa, segundo Köppen).

Para os métodos que tiveram desempenho "bom", com exceção de Blaney-Criddle, notou-se superestimativa da evapotranspiração de referência em relação ao método-padrão $\left(3,91 \mathrm{~mm} \mathrm{~d}^{-1}\right)$, com ETo de 5,26 $\mathrm{mm} \mathrm{d}^{-1}$ para o método de Jensen-Haise e 4,56 $\mathrm{mm} \mathrm{d}^{-1}$ para o da Radiação Solar. Notou-se, também, superestimativas para o método Hargreaves-Samani, com $5,15 \mathrm{~mm} \mathrm{~d}^{-1}$ (Tabela 2). Situação confirmada ao analisar o Erro-Padrão da Estimativa (EPE) em relação ao método-padrão, pois estes foram os métodos que apresentaram os maiores erros.

Na Tabela 2, foi possível verificar que, para escala diária, os métodos que utilizam dados de radiação solar global apresentaram maior desempenho do que aqueles que utilizaram apenas a temperatura do ar. O mesmo comportamento foi observado para a região da Serra da Mantiqueira para os métodos Radiação Solar, Jensen-Haise, Makkink 
e Hargreaves-Samani (PEREIRA et al., 2009).

As estimativas da evapotranspiração na escala mensal, obtidas por diferentes métodos em relação ao método-padrão, para o período de outubro a março, estão representadas na Figura 3, na qual foi possível observar que os métodos Radiação Solar, Camargo, Thornthwaite e Blaney-Criddle melhoram seus coeficientes nessa escala, enquanto para os demais, o desempenho piorou, em relação à escala diária (Tabela 2). Os melhores desempenhos foram para os métodos Radiação Solar, Makkink, Camargo e Blaney-Criddle (Figura 3A, 3D, 3E e 3G).

Ainda, como demonstrado na Figura 3, observou-se que os métodos de Linacre (Figura 3B) e de Makkink (Figura 3D) tenderam a subestimar os valores da ETo, apesar de terem um EPE inferior a $30 \mathrm{~mm} \mathrm{~m}^{-1}$. Desta forma, apresentaram tendência de que, quando aplicados a um balanço hídrico, podem subestimar a demanda hídrica potencial das plantas. Sendo esperado o contrário para os métodos que superestimam a ETo, como o método Radiação Solar, Jensen-Haise e Hargreaves-Samani (Figura 3A, 3C e $3 \mathrm{H})$. Apesar de estes métodos não apresentarem desempenho "péssimo" ou "mau", constata-se que estes não são bons estimadores da ETo, para as condições estudadas, pois o EPE foi de 25,75; 41,66 e $38,20 \mathrm{~mm} \mathrm{~m}^{-1}$, respectivamente.

Porém, para os métodos de Camargo, Thornthwaite e Blaney-Criddle, nota-se que, quando os valores da ETo são baixos, ocorre subestimava ou valores muito próximos ao estimado pelo métodopadrão. Porém, quando apresenta valores maiores, estes acabam sendo superestimados, como observado nas Figuras 3E, 3F e 3G. Para estes métodos e para Linacre, na escala mensal, foram obtidos os menores coeficientes de determinação $\left(\mathrm{R}^{2}\right)$. Mesmo assim, observa-se que, com exceção de Linacre, estes foram os métodos que apresentaram o menor erro-padrão da estimativa, bem como o melhor ajuste 1:1 (Figura 3).

Observou-se que os métodos de Radiação Solar e Camargo apresentaram o maior índice de desempenho $(0,75)$ e EPE de 25,75 e 14,71 mm $\mathrm{m}^{-1}$, respectivamente. Destacam-se, também, os métodos de Makkink e de Blaney-Criddle com desempenho considerado "bom". Assim, levando em consideração o desempenho e o EPE obtido pelos métodos testados, na escala mensal, para a região da Campanha, constata-se que o método de Camargo apresentou os melhores índices $\left(\mathrm{R}^{2}=0,69, \mathrm{~d}=0,91\right.$ e c $=0,75$ ), bem como a melhor relação do tipo $1: 1$ e o menor EPE $\left(14,71 \mathrm{~mm} \mathrm{~m}^{-1}\right)$ (Figura 3E).

Tem-se que o método de Thornthwaite pode super ou subestimar a evapotranspiração em função de não integrar no cálculo a influência das demais variáveis meteorológicas, utilizando apenas a temperatura do ar (CAMARGO; CAMARGO, 2000; CONCEIÇÃO, 2003; JUNGES et al., 2012). No entanto, Camargo e Camargo (2000) afirmam que o modelo de Thornthwaite funciona adequadamente em regiões de clima úmido, independentemente da latitude e da altitude. Além disso, Melo e Fernandes (2012), avaliando métodos empíricos para estimar a evapotranspiração de referência em Uberaba, em Minas Gerais, constataram que métodos que usam apenas temperatura ou radiação solar como variáveis de entrada apresentaram melhor desempenho quando comparados aos métodos que utilizam maior número de variáveis, destacando-se os métodos de Makkink, Camargo, Priestley-Taylor, Hargreaves-Samani e Thornthwaite.

Com base nos valores estimados pelos diferentes métodos, na escala mensal, foram realizadas as comparações para o índice de seca, obtido no mês de março, em relação ao métodopadrão (Figura 4). Desta maneira, verificou-se que os métodos Jensen-Haise (Figura 4C) e Hargreaves-Samani (Figura 4H) foram os métodos que apresentaram maior dispersão do IS, como pode ser observado pela linha de tendência e pelos índices de desempenho. Além disso, estes dois métodos obtiveram elevado erro-padrão da estimativa aplicado ao método do índice de seca (EPE_IS), com valores superiores a $100 \mathrm{~mm} \mathrm{~m}^{-1}$, enquanto nos demais apresentaram EPE abaixo de $40 \mathrm{~mm} \mathrm{~m}^{-1}$. Possivelmente, isto está relacionado diretamente ao fato de estes dois métodos terem constantemente superestimado a evapotranspiração de referência na escala mensal, além de seu alto EPE (Figura 3).

Em uma análise de 81 regiões vitícolas, em 18 países, incluindo Bagé, Conceição et al. (2012) verificaram que o método Hargreaves-Samani, aplicado ao Índice de Seca, em comparação ao método Penman-Monteith, obteve coeficiente de determinação $\left(\mathrm{R}^{2}\right)$, de correlação (r) e índice de concordância (d) de: 0,$80 ; 0,90$ e 0,94, respectivamente. Sendo que o desempenho (c) deste método, para todas as regiões de abrangência do estudo, foi igual a 0,85 . Esses autores concluíram que o método de Hargreaves-Samani pode ser empregado para o cálculo do IS, visando a determinar a classe de clima vitícola do Sistema CCM, apresentando desempenho entre "muito bom" e "ótimo".

Neste estudo, observa-se que o método de Hargreaves-Samani não apresentou ajuste tão bom quanto o encontrado por Conceição et al. (2012), apresentando coeficiente de determinação $\left(\mathrm{R}^{2}\right)$ e índice de concordância (d) de 0,61e 0,58, respectivamente, o que resultou em coeficiente de 
desempenho (c) de 0,46 , classificado como "mau" (Figura 4H). Comparando os resultados deste estudo com os obtidos por Conceição et al. (2012), constata-se a importância de trabalhos regionalizados deste gênero, para validar métodos alternativos ao de Penman-Monteith para a determinação da ETo aplicados ao cálculo do IS.

Na Figura 5, está representado o erro-padrão aplicado ao índice de seca (EPE_IS, $\mathrm{mm} \mathrm{m}^{-1}$ ), para o período de outubro a março e para o mês de março. Observa-se que todos os métodos apresentaram erro-padrão da estimativa maiores no mês de março do que no período de outubro a março, com exceção do método de Makkink, que apresentou EPE_IS menor, e ao de Linacre, com valores muito próximos. Este valor de EPE_IS, no mês de março, justifica-se pelo número de casos analisados, que é menor e mais homogêneo do que o para o período de outubro a março, diminuindo, assim, a variação entre os resultados obtidos. Para Makkink e Linacre, os resultados obtidos estão relacionados diretamente ao fato de estes subestimarem os valores da ETo; logo, estavam sempre próximos à capacidade do campo $(200 \mathrm{~mm})$.

Também na Figura 5, constata-se que o erropadrão aplicado ao índice de seca (EPE_IS) confirma o desempenho dos métodos aplicados ao índice de seca (Figura 4), já que os métodos apresentaram erro proporcional ao desempenho obtido. Assim, os métodos de Jensen-Haise, Hargreaves-Samani e Radiação Solar apresentaram os maiores erros.

Destaca-se que os demais métodos aplicados ao IS apresentaram EPE_IS, no período de outubro a março, inferior a $23 \mathrm{~mm} \mathrm{~m}^{-1}$ (ETo_LI), e para março, inferior a $25 \mathrm{~mm} \mathrm{~m}^{-1}$. Por terem apresentado erros relativamente baixos, os métodos de Linacre, Makkink, Camargo, Thornthwaite e Blaney-Criddle apresentam-se como opções viáveis para estimar a ETo, na região da Campanha.

Deve-se tomar cuidado especial em relação aos métodos de Linacre e Makkink (Figura 4), que acabam subestimando os valores da ETo e, quando aplicados ao IS, resultaram em valores de disponibilidade hídrica próximo a $200 \mathrm{~mm}$, indicando clima vitícola da classe Úmido. Tal resultado pode ser verificado para os métodos de Linacre e Makkink, que apresentaram as menores médias e somatórios da ETo (Tabela 3), sendo que, para o período de outubro a março, em função dos baixos valores obtidos da evapotranspiração, o IS mensal foi sempre igual a $200 \mathrm{~mm}$.

De forma geral, notou-se que todos os municípios apresentaram a mesma tendência quando comparados com o valor médio dos municípios da região da Campanha. Assim, os métodos de Jensen-Haise e Hargreaves-Samani foram os que resultaram nas maiores médias e do somatório da ETo, consequentemente, nos menores valores do IS (Tabela 3).

O método de Jensen-Haise obteve, para todos os municípios estudados da Campanha, uma classificação para o IS em relação ao método de Penman-Monteith. O mesmo também foi observado para o método de Hargreaves-Samani para Alegrete, Bagé e São Gabriel (Tabela 3).

Outro método que obteve valores altos da estimativa da ETo foi o da Radiação Solar, nos seis municípios da região estudada, mas a classificação para o IS foi a mesma que o método-padrão do Sistema CCM obteve (Tabela 3).. Porém, tal método merece ressalva, principalmente em anos com baixos volumes de precipitação, pois foi o terceiro método com maior erro na estimativa aplicado ao índice de seca; logo, podendo apresentar uma classificação diferente da obtida pelo método-padrão (Figura 5).

Os métodos que, aplicados ao IS, apresentaram os valores mais próximos aos obtidos pelo métodopadrão foram Camargo e Thornthwaite, para todos os municípios abrangidos neste estudo, na região da Campanha (Tabela 3). Desta forma, tais métodos apresentam maior desempenho e precisão na estimativa da evapotranspiração para a determinação do índice de seca, em relação ao método-padrão (Penman-Monteith), dentre os métodos analisados. 


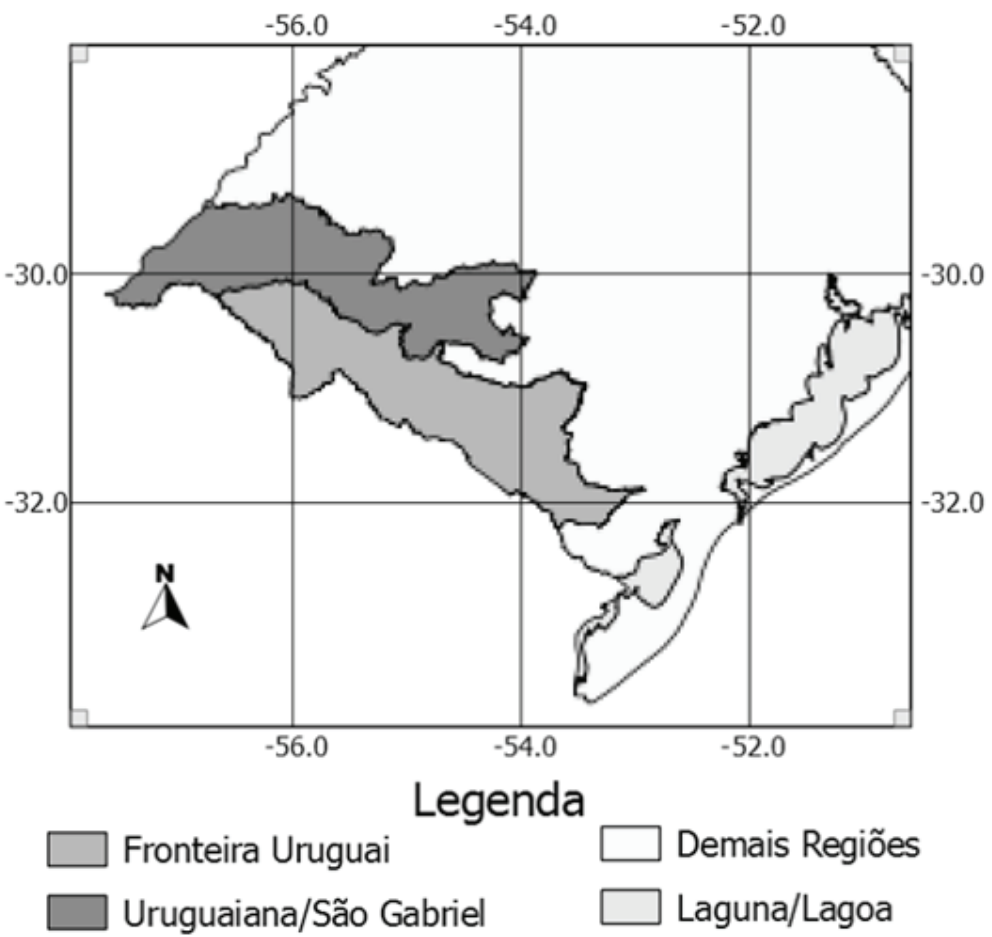

FIGURA 1 - Região da Campanha do Rio Grande do Sul (RS), dividida em duas sub-regiões: Fronteira Uruguai e Uruguaiana/São Gabriel, conforme descrito no macrozoneamento agroecológico e econômico do estado do Rio Grande do Sul.
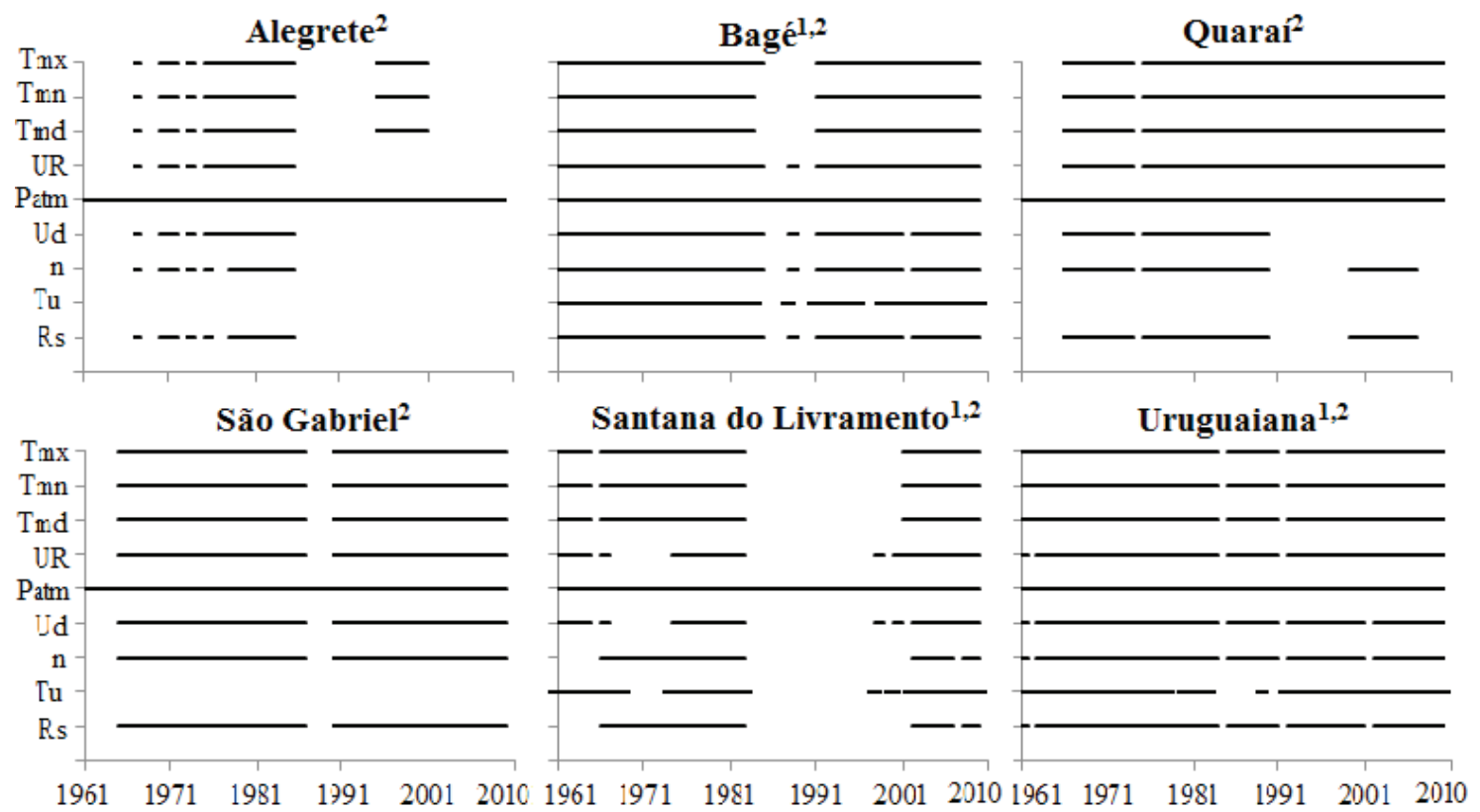

FIGURA 2 - Disponibilidade de dados de precipitação (P), temperatura máxima (Tmx), temperatura mínima (Tmn), temperatura média (Tmd), umidade relativa (UR), pressão atmosférica (Patm), velocidade média do vento (Ud), insolação (n), temperatura de bulbo úmido $(\mathrm{Tu})$, e radiação solar global (Rs), nas estações meteorológicas nos municípios da região da Campanha, RS, para o período de outubro a março, para os anos de 1961 a 2010. ${ }^{1}$ INMET, ${ }^{2}$ FEPAGRO. 


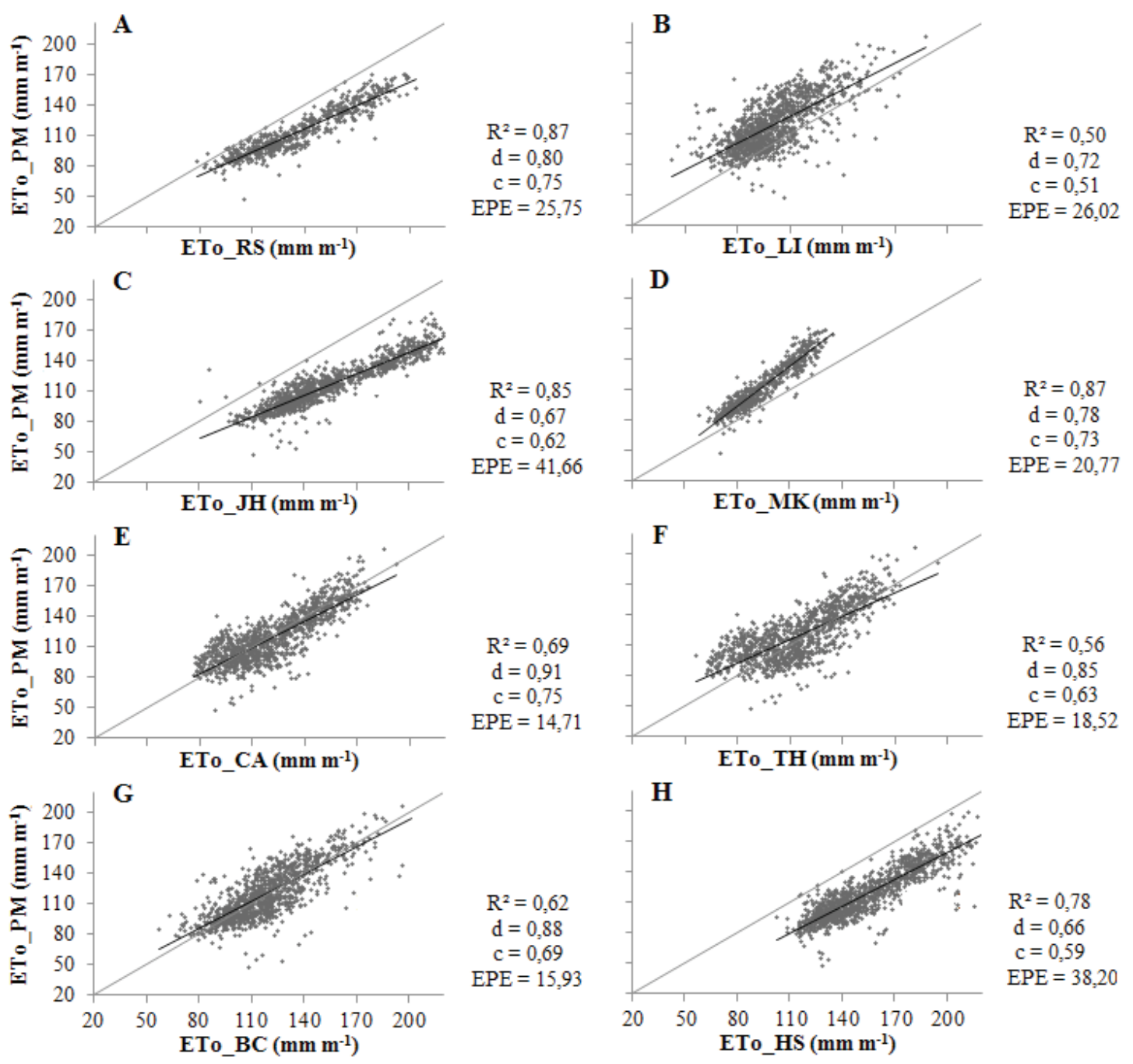

FIGURA 3 - Evapotranspiração de referência na escala mensal (ETo, $\mathrm{mm} \mathrm{m}^{-1}$ ), estimado pelo método de Penman-Monteith (ETo_PM), em relação aos métodos de Radiação Solar (ETo_RS) (A), Linacre (ETo_LI) (B), Jensen-Haise (ETo_JH) (C), Makkink (ETo_MK) (D), Camargo (ETo_CA) (E), Thornthwaite (ETo_TH) (F), Blaney-Criddle (ETo_BC) (G) e HargreavesSamani (ETo_HS) (H), para o período de outubro a março, nos anos de 1961 a 2010, na região da Campanha-RS. Índice de determinação $\left(\mathrm{R}^{2}\right)$, de exatidão (d), de desempenho (c) e Erro-Padrão da Estimativa (EPE, $\left.\mathrm{mm} \mathrm{m}^{-1}\right)$. 

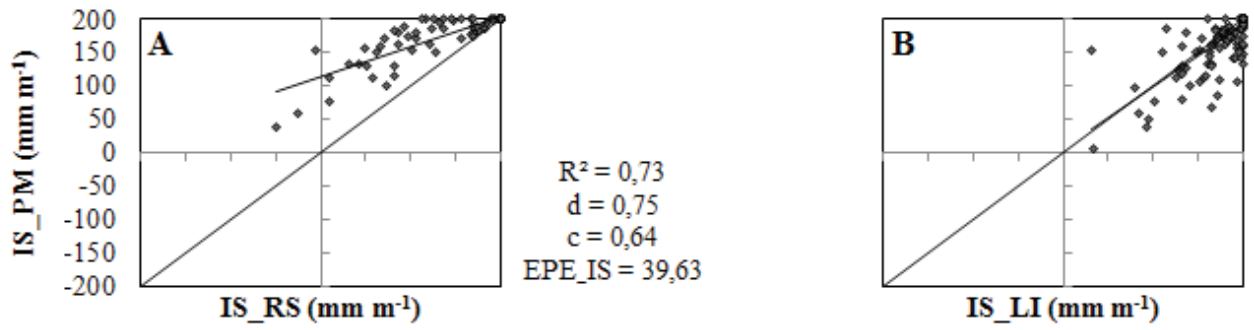

$\mathrm{R}^{2}=0,59$

$\mathrm{d}=0,86$

$\mathrm{c}=0,66$

IS_LI $\left(\mathrm{mm} \mathrm{m}^{-1}\right)$

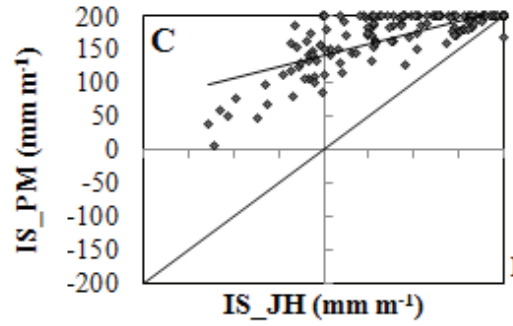

$R^{2}=0,59$

$\mathrm{d}=0,55$

$\mathrm{c}=0,42$ EPE_IS $=138,19$

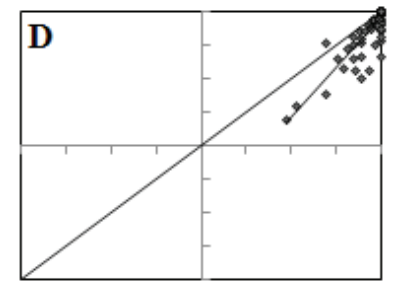
EPE_IS $=23,71$
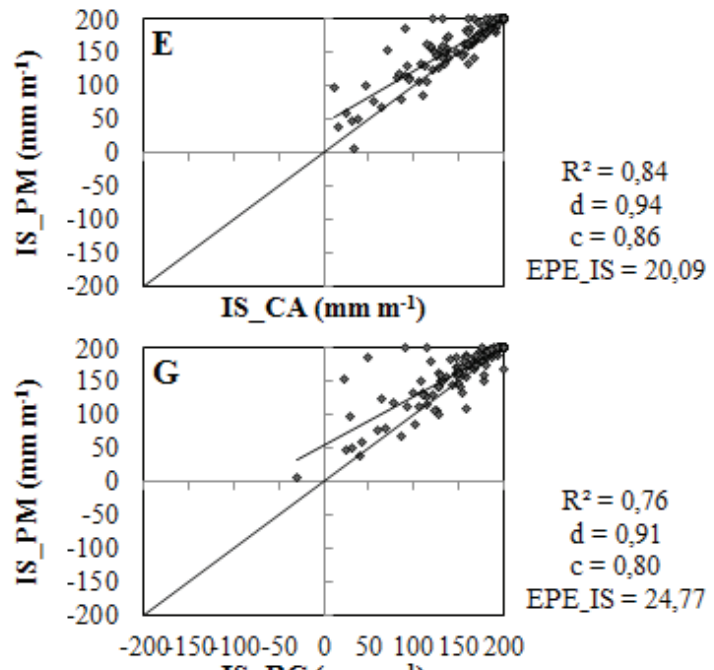

$$
\begin{gathered}
\mathrm{R}^{2}=0,76 \\
\mathrm{~d}=0,91 \\
\mathrm{c}=0,80 \\
\text { EPE_IS }=24,77
\end{gathered}
$$

IS_BC $\left(\mathrm{mm} \mathrm{m}^{-1}\right)$

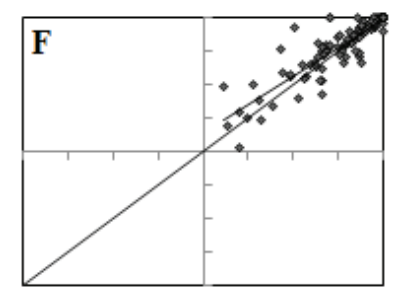

IS TH $\left(\mathrm{mm} \mathrm{m}^{-1}\right)$

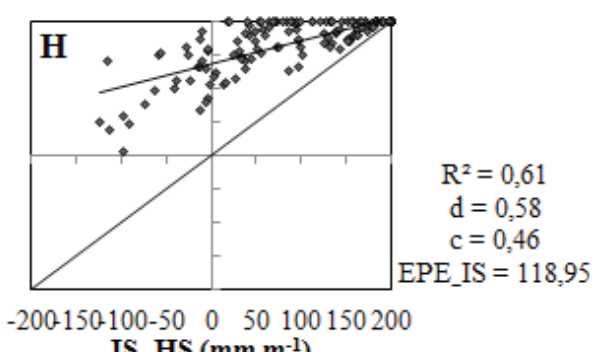

IS_HS $\left(\mathrm{mm} \mathrm{m}^{-1}\right)$

FIGURA 4 - Índice de Seca $\left(\mathrm{mm} \mathrm{m}^{-1}\right)$ determinado com base no método de Penman-Monteith (IS PM), em relação aos métodos da Radiação Solar (IS_RS) (A), Linacre (IS_LI) (B), Jensen-Haise (IS_JH) (C), Makkink (IS_MK) (D), Camargo (IS_CA) (E), Thornthwaite (IS_TH) (F), Blaney-Criddle (IS_BC) (G) e Hargreaves-Samani (IS_HS) (H), para o mês de março, nos anos de 1961 a 2010, para a região da Campanha-RS. Índice de determinação $\left(\mathrm{R}^{2}\right)$, de exatidão (d), de desempenho (c) e Erro Padrão da Estimativa do Índice de Seca (EPE_IS, $\mathrm{mm} \mathrm{m}^{-1}$ ). 


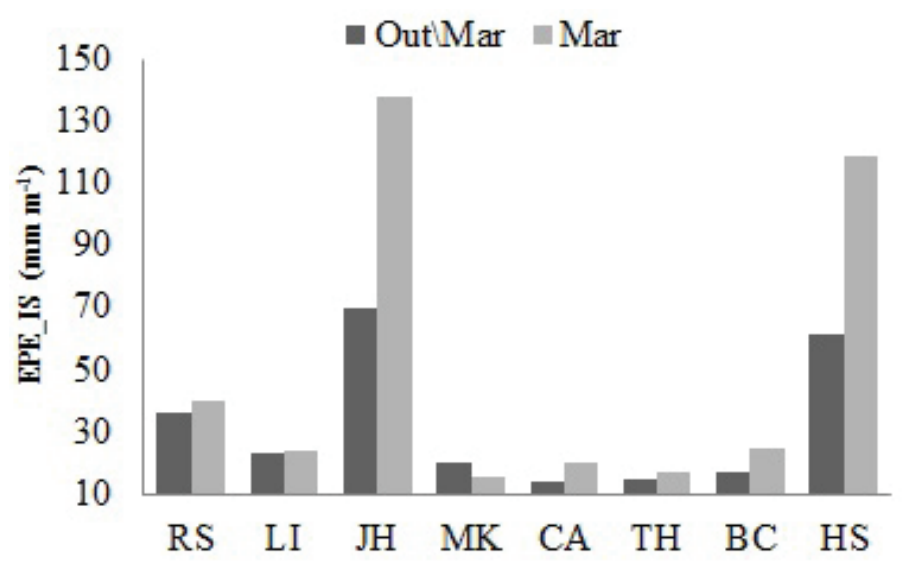

FIGURA 5 - Erro-padrão na determinação do Índice de Seca (EPE_IS, $\mathrm{mm} \mathrm{m}^{-1}$ ), com base no período de outubro a março (OutlMar) e para o mês de março (Mar), para os métodos Radiação Solar (RS), Linacre (LI), Jensen-Haise (JH), Makkink (MK), Camargo (CA), Thornthwaite (TH), Blaney-Criddle (BC) e Hargreaves-Samani (HS), em comparação ao de Penman-Monteith, na região da Campanha-RS, nos anos de 1961 a 2010.

TABELA 1 - Classes do clima vitícola para o Índice de Seca (IS, $\mathrm{mm}$ ), com respectiva simbologia e intervalo da classe, conforme proposto por Tonietto e Carbonneau (2004).

\begin{tabular}{lcc}
\hline \multicolumn{1}{c}{ Classes do clima } & Sigla & Intervalo de classe \\
\hline Úmido & IS-2 & $150<$ IS \\
Subúmido & IS-1 & $50<$ IS $\leq 150$ \\
De seca moderada & IS +1 & $-100<$ IS $\leq 50$ \\
De seca forte & IS +2 & IS $\leq-100$ \\
\hline
\end{tabular}

TABELA 2 - Média da evapotranspiração de referência calculada na escala diária (ETo, $\left.\mathrm{mm} \mathrm{d}^{-1}\right)$, erro-padrão, estimativa (EPE, $\left.\mathrm{mm} \mathrm{d}^{-1}\right)$, coeficientes de determinação $\left(\mathrm{R}^{2}\right)$, de precisão $(\mathrm{r})$, de exatidão $(\mathrm{d})$ e de desempenho (c) da comparação e classificação entre o método- padrão com os demais métodos de estimativa da evapotranspiração, para a região da Campanha-RS, para os meses de outubro a março, com base no período de 1961 a 2010.

\begin{tabular}{cccccccccc}
\hline & ETo_RS & ETo_LI & ETo_JH & ETo_MK ETo_CA & ETo_TH & ETo_BC & ETo_HS & ETo_PM \\
\hline ETo $\left(\mathrm{mm} \mathrm{d}^{-1}\right)$ & 4,56 & 3,31 & 5,26 & 3,19 & 4,04 & 3,80 & 3,89 & 5,15 & 3,91 \\
EPE $\left(\mathrm{mm} \mathrm{d}^{-1}\right)$ & 1,13 & 1,15 & 1,56 & 0,94 & 1,00 & 1,06 & 0,85 & 1,47 & - \\
$\mathrm{R}^{2}$ & 0,70 & 0,51 & 0,77 & 0,69 & 0,47 & 0,43 & 0,65 & 0,61 & - \\
r & 0,84 & 0,71 & 0,78 & 0,83 & 0,69 & 0,66 & 0,81 & 0,78 & - \\
d & 0,84 & 0,78 & 0,78 & 0,82 & 0,81 & 0,80 & 0,90 & 0,74 & - \\
c & 0,70 & 0,56 & 0,68 & 0,78 & 0,56 & 0,53 & 0,72 & 0,58 & - \\
Clasf* & BM & SF & BM & MB & SF & SF & BM & SF & - \\
\hline
\end{tabular}

Radiação Solar (ETo_RS), Linacre (ETo_LI), Jensen-Haise (ETo_JH), Makkink (ETo_MK), Camargo (ETo_CA), Thornthwaite (ETo_TH), Blaney-Criddle (ETo_BC), Hargreaves-Samani (ETo_HS) e Penman-Monteith (ETo_PM).

* Classificação: MB: muito bom; BM: bom; SF: sofrível. 
을 :

$\exists$

응 है을

产

음 0 可

e ${ }^{\prime}$ :

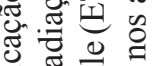

政

诺

융 交㽦

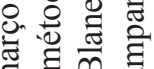

记

退

है

可近

\&

娄

芯 范

의

䒕

हี

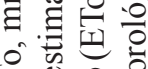

ป 일

त

ए

है

¿ $\sum$

ष잉

怘氙苛

$\exists$ 递

के

in 0

至島

讨 0 .

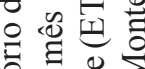

웅욜

퓰 坖

0 10

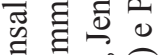

包告

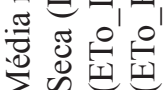

乘

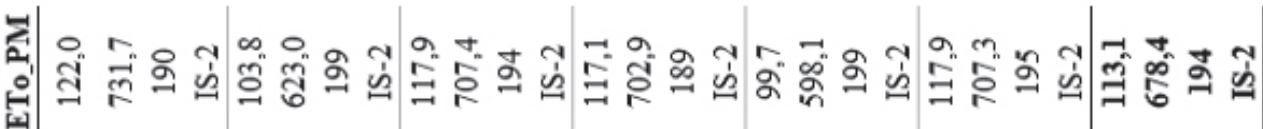

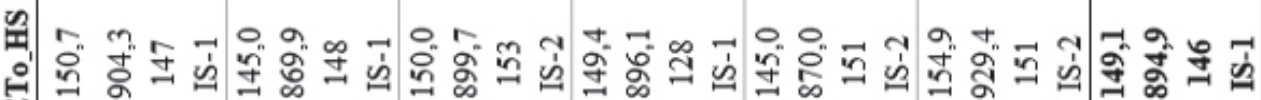

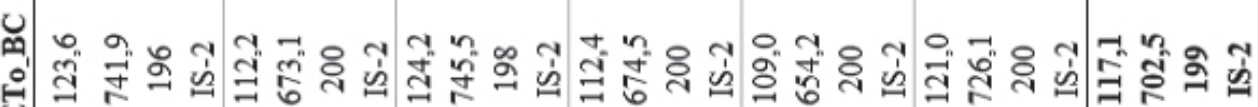

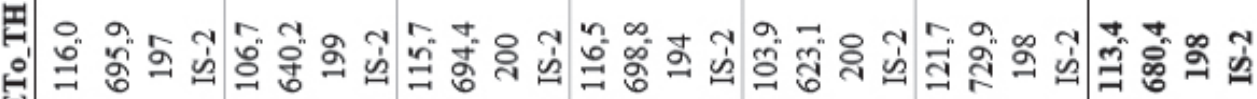

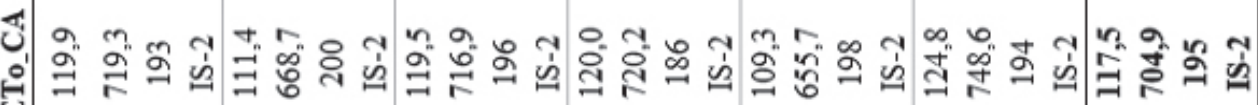

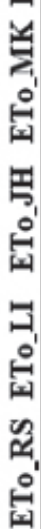

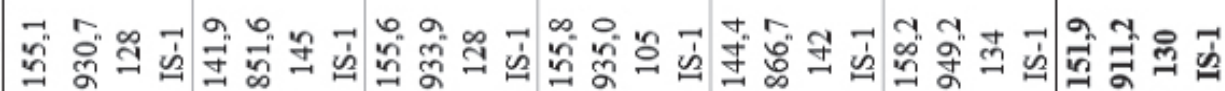

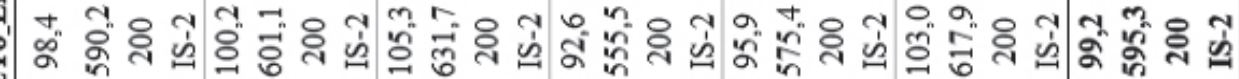

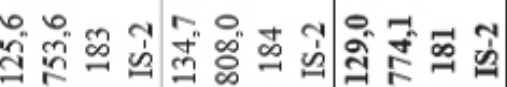

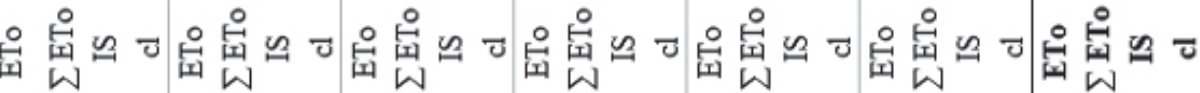

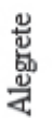
范 筧 


\section{CONCLUSÕES}

Para as condições climáticas da região da Campanha, na escala diária, no período de outubro a março, o melhor ajuste, dentre os métodos estudados, é para o método Makkink, que apresenta desempenho "muito bom" para estimar a evapotranspiração, quando comparado ao método-padrão (PenmanMonteith). Na escala mensal, os métodos que se destacam pelo seu desempenho são: Radiação Solar, Makkink, Camargo e Blaney-Criddle, com desempenho "bom".

Os métodos de Thornthwaite e Camargo apresentam desempenho "ótimo" para estimar a evapotranspiração de referência aplicados ao índice de seca (IS). Desta forma, estes métodos podem ser empregados ao IS quando não se dispõe de dados meteorológicos para cálculo da ETo pelo métodopadrão, para as condições climáticas da Campanha do Rio Grande do Sul.

Os métodos de Jensen-Haise e HargreavesSamani não são metodologias recomendadas para cálculo da ETo, para determinar o índice de seca, nas condições climáticas da Campanha-RS, pois resultam em classificação do clima vitícola para o IS diferente da obtida pelo método-padrão do Sistema CCM Geovitícola.

\section{AGRADECIMENTO}

À Fundação Estadual de Pesquisa Agropecuária Gaúcha (FEPAGRO) e ao Instituto Nacional de Meteorologia - $8^{\circ}$ Distrito de Meteorologia (INMET $-8^{\circ}$ DISME), pela disponibilidade dos dados meteorológicos, fundamentais para a realização deste trabalho.

\section{REFERÊNCIAS}

AMARAL, do U.; MARTINS, C. R.; COELHO FILHO, R.; BRIXNER, G. F.; BINI, D. A. Caracterização fenológica e produtiva de videiras Vitis vinifera L. cultivadas em Uruguaiana e Quaraí-RS. Revista da FZVA, Uruguaiana, v.16, n.1, p. 22-31. 2009. Disponível em: $\leq$ http:// revistaseletronicas.pucrs.br/ojs/index.php/fzva/ article/viewFile/3625/4162>. Acesso em: 20 ago.2012.
BORGES JÚNIOR, J. C. F.; ANJOS, R. J.; SILVA, T. J. A.; LIMA, J. R. S.; ANDRADE, C. L. T. Métodos de estimativa da evapotranspiração de referência diária para a microrregião de Garanhuns-PE. Revista Brasileira de Engenharia Agrícola e Ambiental, Campina Grande, v.16, n.4, p.380-390, 2012. Disponível em: <http://www.scielo.br/pdf/rbeaa/ v16n4/v16n04a08.pdf>. Acesso em : 26 ago. 2013.

CAMARGO, A. P.; CAMARGO, M. B. P. Uma revisão analítica da evapotranspiração potencial. Bragantina, Campinas, v.59, n.2, p.125-137, 2000. Disponível em: <http://www.scielo.br/pdf/brag/ v59n2/a02v59n2.pdf>. Acesso em: 20 out. 2012.

CARVALHO, L. G.; RIOS, G. F. A.; MIRANDA, W. L.; NETO, P. C. Evapotranspiração de referência: Uma abordagem atual de diferentes métodos de estimativa. Pesquisa Agropecuária Tropical, Goiânia, v.41, n.3, p.456-465, 2011. Disponível em: $<$ http://www.revistas.ufg.br/index.php/pat/article/ view/12760/9732>. Acesso em: 10 out. 2012.

CONCEIÇÃO, M. A. F. Estimativa da evapotranspiração de referência com base na temperatura do ar para as condições do Baixo Rio Grande-SP. Revista Brasileira deAgrometeorologia, Santa Maria, v.11, n.2, p.229-236, 2003.

CONCEIÇÃO, M. A. F.; TONIETTO, J.; FIALHO, F. B. Uso da temperatura para cálculo do índice de seca de regiões produtoras de uva. Revista Brasileira de Fruticultura, Jaboticabal, v.34, n.1, p.175-182, 2012. Disponível em: $\leq$ http://www.scielo.br/pdf/rbf/ v34n1/v34n1a24.pdf>. Acesso em: 11 ago. 2012.

FONTANA, D. C.; OLIVEIRA, D. Relação entre radiação solar global e insolação para o Estado do Rio Grande do Sul. Revista Brasileira de Agrometeorologia, Santa Maria, v.4, n.1, p.87-91, 1996.

JUNGES, T. S. C.; DALLACORT, R.; INOUE, M. H.; MODOLO, A. J.; RAMOS, H. da C. Estimativa da evapotranspiração de referência por diferentes métodos para a região de Sinop-MT. Enciclopédia Biosfera, Goiânia, v.8, n.14, p.516-526, 2012. Disponível em: <http://www.conhecer.org.br/ enciclop/2012a/agrarias/estimativa.pdf $>$. Acesso em: 30 out. 2012 .

LIU, X.; MEI, X.; LI, Y.; WANG, Q.; ZHANG, Y.; PORTER, J. R. Variation in reference crop evapotranspiration caused by the Angström- 
Prescott coefficient: Locally calibrated versus the FAO recommended. Agricultural Water Management, Columbus, v.96, n.6 p.1137-1145, 2009. Disponível em: $<$ http://dx.doi.org/10.1016/j. agwat.2009.03.005 >. Acesso em: 18 mar. 2014.

MELO, G. de; FERNANDES, A. L. T. Evaluation of empirical methods to estimate reference evapotranspiration in Uberaba, State of Minas Gerais, Brazil. Revista da Engenharia Agrícola, Jaboticabal, v.32, n.5, p.875-888, 2012. Disponível em: $<$ http://www.scielo.br/pdf/eagri/v32n5/07.pdf $>$. Acesso em: 20 dez. 2012.

PEREIRA, D. dos R.; YANAGI, S. de N. M.; MELLO, C. R. de; SILVA, A. M. da; SILVA, L. A. da S. Desempenho de métodos de estimativa da evapotranspiração de referência para a região da Serra da Mantiqueira-MG. Ciência Rural, Santa Maria, v.39, n.9, p.2.488-2.493, 2009. Disponível em: $\leq$ http://www.scielo.br/pdf/cr/v39n9/a376cr1581. pdf $>$. Acesso em: 21 set. 2012.

PILAU, F. G.; BATTISTI, R.; SOMAVILLA, L.; RIGHI, E. Z. Desempenho de métodos de estimativa da evapotranspiração de referência nas localidades de Frederico Westphalen e Palmeira das Missões-RS. Ciência Rural, Santa Maria, v.42, n.2, p.283-290, 2012. Disponível em: <http://www.scielo.br/pdf/cr/ v42n2/a5412cr5346.pdf> . Acesso em: 26 ago. 2013.

PÖTTER, G. H.; DAUTL, C. E.; BRACKAMNNL, A.; LEITE, T. T.; PENNA, N. G. Desfolha parcial em videiras e seus efeitos em uvas e vinhos Cabernet Sauvignon da região da Campanha do Rio Grande do Sul, Brasil. Ciência Rural, Santa Maria, v.40, n.9, p.2011-2016, 2010. Disponível em: <http://www. scielo.br/pdf/cr/v40n9/a723cr2379.pdf $>$. Acesso em: 21 ago. 2013.
RATHMANN, R; HOFF, D. N.; SANTOS, O. I. B.; PADULA, A. D. Diversificação Produtiva e como Possibilidades de Desenvolvimento: um Estudo da fruticultura na Região da Campanha. Revista Economia Social Rural, v.46, n.2 p.325354, 2008. Disponível em: <http://www.scielo. br/scielo.php?script $=$ sci arttext\&pid $=$ S0103$20032008000200003 \& \operatorname{lng}=$ en $\& n r m=$ iso $>$. ISSN 0.103-2.003.http://dx.doi.org/10.1590/S010320032008000200003 . Acessado em: 10 out. 2011.

SILVA, V. J. da; CARVALHO, H. de P.; DA SILVA, C. R.; CAMARGO, R. de; TEODORO, R. E. F. Desempenho de diferentes métodos de estimativa da evapotranspiração de referência diária em Uberlândia-MG. Bioscience Journal, Uberlândia, v. 27, n.1, p.95-101, 2011. Disponível em: $<\underline{\text { http: } / /}$ www.seer.ufu.br/index.php/biosciencejournal/ article/view/7374/6846>. Acesso em: 13 mar. 2014.

TONIETTO, J.; CARBONNEAU, A. A multicriteria climatic classification system for grape-growing regions worldwide. Agricultural and Forest Meteorology, Amsterdam, v.124, n.1-2, p.81-97, 2004. Disponível em: $<\underline{\text { http://www.sciencedirect. }}$ com/science/article/pii/S0168192304000115>. Acesso em: 10 maio 2012.

TONIETTO, J.; FIALHO, F. B. The geoviticulture MCC system and its international internet site. In: TONIETTO, J.; RUIZ, V. S.; GÓMEZ-MIGUEL, V. D. (Org.) Clima, zonificación y tipicidad del vino en regiones vitivinícolas ibero-americanas. Madri, 2012. Cap. 1 . p. 21-38. 